\title{
Review
}

Kidney Dis

DOI: 10.1159/000487671
Received: January 11, 2018

Accepted after revision: February 14, 2018

Published online: April 3, 2018

\section{Novel Insights into Crystal-Induced Kidney Injury}

\author{
Shrikant R. Mulay Chongxu Shi Xiaoyuan Ma Hans Joachim Anders \\ Division of Nephrology, Medizinische Klinik und Poliklinik IV, Klinikum der Universität München, LMU Munich, \\ Munich, Germany
}

\section{Keywords}

Acute kidney injury · Chronic kidney disease · Fibrosis .

Inflammation - Tubulointerstitial nephritis

\begin{abstract}
Background: The entity of crystal nephropathies encompasses a spectrum of different kidney injuries induced by crystal-formed intrinsic minerals, metabolites, and proteins or extrinsic dietary components and drug metabolites. Depending on the localization and dynamics of crystal deposition, the clinical presentation can be acute kidney injury, progressive chronic kidney disease, or renal colic. Summary: The molecular mechanisms involving crystal-induced injury are diverse and remain poorly understood. Type 1 crystal nephropathies arise from crystals in the vascular lumen (cholesterol embolism) or the vascular wall (atherosclerosis) and involve kidney infarcts or chronic ischemia, respectively. Type 2 crystal nephropathies arise from intratubular crystal deposition causing obstruction, interstitial inflammation, and tubular cell injury. NLRP3 inflammasome and necroptosis drive renal necroinflammation in acute settings. Type 3 is represented by crystal and stone formation in the draining urinary tract, i.e., urolithiasis, causing renal colic and chronic obstruction. Key Messages: Dissecting the types of injury is the first step towards a better understanding of the patho-
\end{abstract}

\section{KARGER}

(c) 2018 S. Karger AG, Basel

E-Mail karger@karger.com

www.karger.com/kdd physiology of crystal nephropathies. Crystal-induced activation of the inflammasome and necroptosis, crystal adhesion, crystallization inhibitors, extratubulation, and granuloma formation are only a few of certainly many involved pathomechanisms that deserve further studies to eventually form the basis for innovative cures for these diseases.

(c) 2018 S. Karger AG, Basel

\section{Introduction}

A substantial number of kidney diseases involve crystalline microparticles that contribute to mechanical obstructions, local intrarenal inflammation, and tissue injury. Crystalline nephropathies can be divided into three subgroups [1]: renal ischemic due to vascular calcifications or crystal embolism (type 1), intra- and extratubular crystalline precipitates causing tubular injury (type 2), and nephrolithiasis causing obstructive nephropathy (type 3) (Fig. 1). Traditionally, research on the pathophysiology of crystalline nephropathies has focused on the physicochemical properties of urinary minerals, supersaturation, and crystal growth inhibition. Accumulating data now enrich these concepts by mechanisms of intrarenal crystal adhesion, crystal-induced inflammation, and cell death that link local crystal deposits to a large 
Fig. 1. The three types of crystalline nephropathies. Type 1 causes mostly acute renal infarcts (e.g., cholesterol embolism) or chronic ischemia (e.g., atherosclerotic renal artery stenosis). Type 2 causes mostly tubular injury either directly by inducing necroptosis or apoptosis by tubular cells or indirectly by inducing inflammation or tubular obstruction. Type 3 is represented by urolithiasis that causes either renal colic or chronic obstructive nephropathy. Each of the classes can involve several types of crystals the most prevalent of which are depicted by scanning electron microscopy. Crystal size is usually in the micrometer range except for cysteine, which has larger sizes.

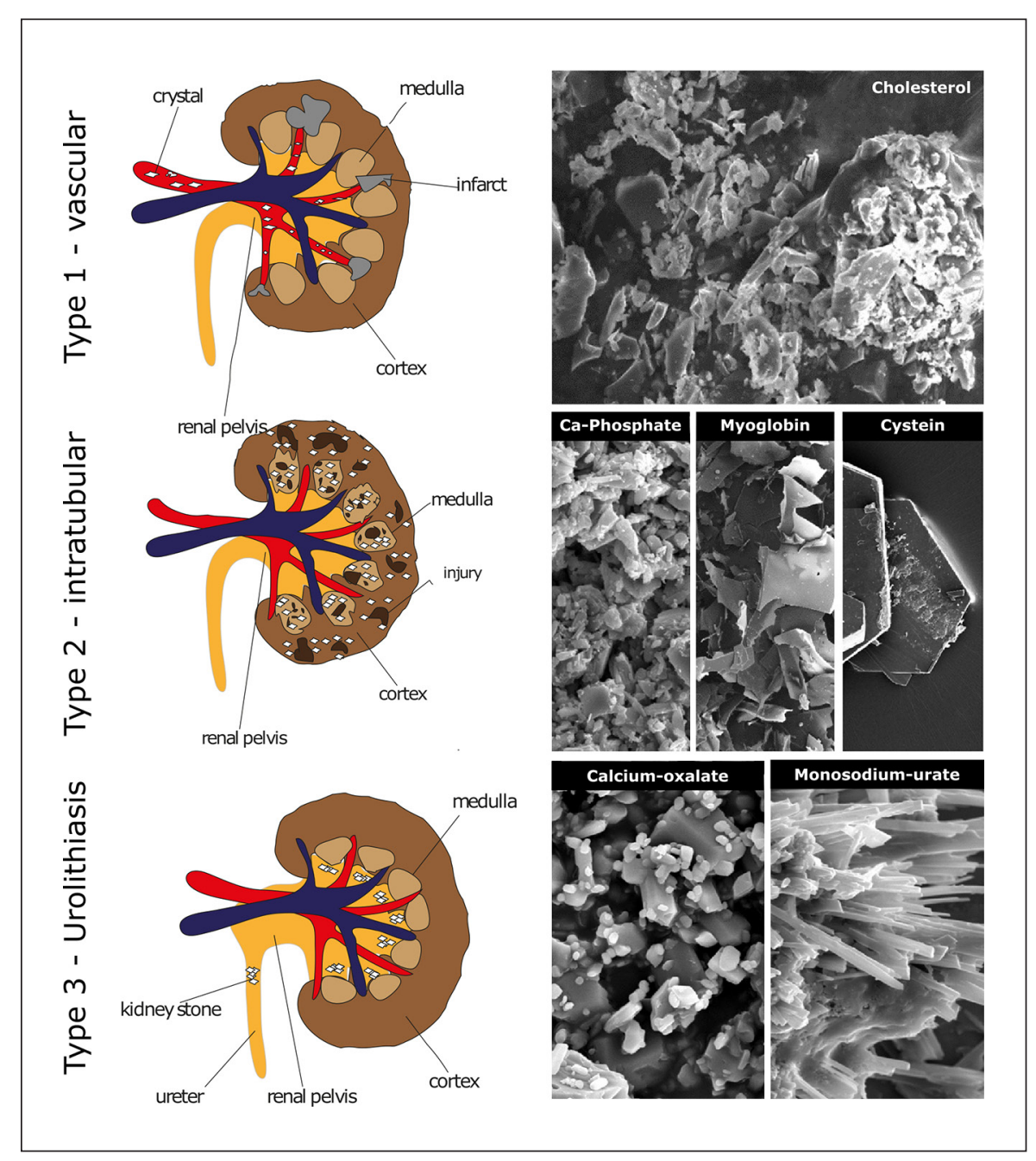

number of different diseases in and outside the kidney referred to as "crystallopathies" [2]. Here, we briefly summarize the clinical presentations and pathophysiological mechanisms of such crystalline nephropathies.

\section{Type 1 Crystalline Nephropathy: Renal Cholesterol Embolism}

Cholesterol crystal embolism (CCE) occurs when emboli of cholesterol crystals (CCs) arising from the rupture of an atherosclerotic plaque from aortic or other major arteries block smaller arteries (150-200 $\mu \mathrm{m}$ in diameter), which may affect kidneys, skin, gastrointestinal system, eyes, muscles, bones, brain, nerves, internal organs, and limbs [3]. Since kidneys are located near the abdominal aorta, they are the most frequent target organ of CCE [3].
However, diagnosis of renal CCEs is difficult and controversial and often based on the presence of a precipitating event, renal failure, and peripheral CCE. Clinically, renal CCE can be acute, subacute, or chronic [3].

A critical factor in the development of renal CCEs is the formation of an atherosclerotic lesion. Spontaneous or iatrogenic rupture of plaques in the thoracic aorta or renal arteries results in showers of CCs, which obstruct the smaller arteries. In the kidney, obstruction of arcuate and interlobar arteries by CCs causes cortical and medullary infarcts [1]. CCs can also induce either passive (physical) or regulated necrosis of the endothelial cells. They are reported to induce endothelial cell injury via complement activation [4]. Furthermore, they kill tubular epithelial cells by activating receptor-interacting serine/ threonine-protein kinase 3 (RIPK3) and mixed lineage kinase domain-like (MLKL) $[5,6]$. Activation of MLKL 
Table 1. Acute or chronic crystal-induced kidney diseases

\begin{tabular}{|c|c|c|c|}
\hline Type & $\begin{array}{l}\text { Renal } \\
\text { damage }\end{array}$ & Disease & Crystal (source of crystal-forming factor) \\
\hline \multirow{2}{*}{$\begin{array}{l}\text { Type } 1 \\
\text { (vascular) }\end{array}$} & AKI & cholesterol embolism & cholesterol \\
\hline & CKD & atherosclerotic renal artery stenosis & cholesterol, apatite, $\mathrm{CaP}$ \\
\hline \multirow{11}{*}{$\begin{array}{l}\text { Type } 2 \\
\text { (tubular) }\end{array}$} & \multirow[t]{6}{*}{ AKI } & \multirow[t]{2}{*}{ diet-induced crystal nephropathy } & $\mathrm{CaOx}$ monohydrate (whewellite) \\
\hline & & & $\mathrm{CaP}_{-}, \mathrm{CaOx}-$, and vitamin C-rich foods and drinks \\
\hline & & $\begin{array}{l}\text { drug-induced crystal nephropathy } \\
\text { tumor lysis syndrome } \\
\text { drug induced crystalluria } \\
\text { acute phosphate nephropathy }\end{array}$ & $\begin{array}{l}\text { uric acid } \\
\text { drug crystals } \\
\mathrm{CaP}\end{array}$ \\
\hline & & myogen crystal nephropathy & myoglobin (rhabdomyolysis) \\
\hline & & bilirubin crystal nephropathy (bile cast nephropathy) & bile pigment \\
\hline & & $\begin{array}{l}\text { paraprotein-induced crystal nephropathy (light-chain cast } \\
\text { nephropathy) }\end{array}$ & monoclonal light chains \\
\hline & CKD & $\begin{array}{l}\text { CAKUT } \\
\text { genetic forms of RTA } \\
\text { genetic forms of hyperuricosuria } \\
\text { primary hyperoxaluria } \\
\text { cystinosis } \\
\text { APRT deficiency }\end{array}$ & $\begin{array}{l}\text { brushite, } \mathrm{CaCO}_{3}, \mathrm{CaOx}, \mathrm{CaP} \text {, hydroxyapatite, struvite } \\
\text { uric acid } \\
\text { CaOx monohydrate (whewellite), CaOx dihydrate } \\
\text { (weddellite) } \\
\text { cystine } \\
\text { adenine }\end{array}$ \\
\hline & & diet- or drug-induced crystal nephropathy & uric acid (diets rich in sodium, fructose, sucrose) \\
\hline & & tophaceous gout & $\begin{array}{l}\text { uric acid (diets low in potassium, calcium, phytate, fluid } \\
\text { administration as part of Mesoamerican nephropathy } \\
\text { treatment) }\end{array}$ \\
\hline & & $\begin{array}{l}\text { immunoglobulin-induced crystal nephropathies } \\
\text { light-chain cast nephropathy, proximal tubulopathy with } \\
\text { or without Fanconi syndrome, crystalglobulinemia, } \\
\text { crystal-storing histiocytosis }\end{array}$ & monoclonal light chains \\
\hline & & $\begin{array}{l}\text { chronic glomerular crystal nephropathies } \\
\text { cryoglobulinemia } \\
\text { crystalglobulinemia } \\
\text { cystinosis }\end{array}$ & $\begin{array}{l}\text { monoclonal and polyclonal IgG } \\
\text { monoclonal light chains } \\
\text { cystine }\end{array}$ \\
\hline
\end{tabular}


Table 1 (continued)

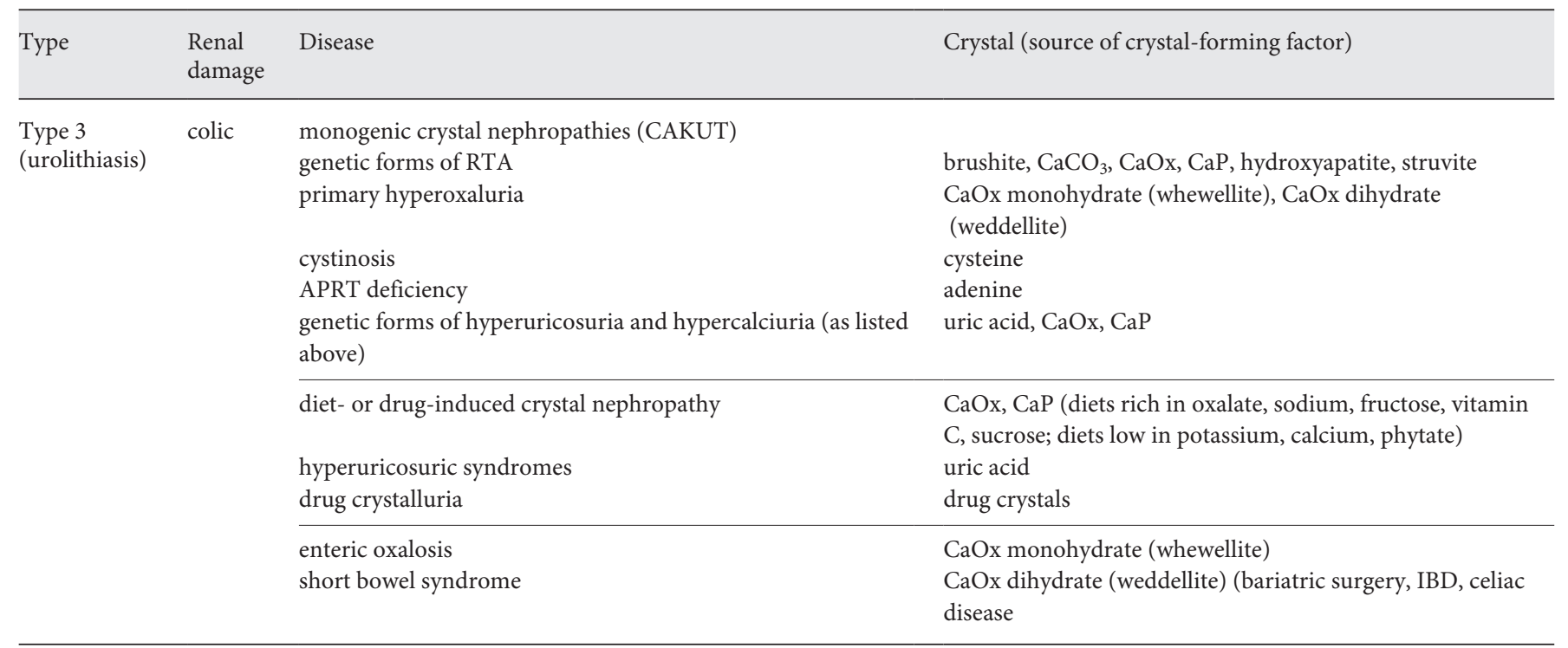

AKI, acute kidney injury; APRT, adenine phosphoribosyl transferase; $\mathrm{CaCO}_{3}$, calcium carbonate; CAKUT, congenital anomalies of the kidney and the urinary tract; $\mathrm{CaOx}$, calcium oxalate; $\mathrm{CaP}$, calcium phosphate; $\mathrm{CKD}$, chronic kidney disease; $\mathrm{IBD}$, inflammatory bowel disease; RTA, renal tubular acidosis.

by RIPK3 results in disruption of the plasma membrane and a regulated form of necrosis, which is termed necroptosis [7]. In addition, CCs can directly induce inflammation. For example, during the development of atherosclerosis, macrophages engulf small CCs, which activate the NLRP3 inflammasome, and thus, set up IL- $1 \beta$-dependent inflammation [8]. The binding of CCs to the human macrophage-inducible C-type lectin receptors on the surface of macrophages and dendritic cells induces secretion of proinflammatory cytokine tumor necrosis factor (TNF) and macrophage inflammatory protein 2 [9]. CCs can also activate Syk and PI3K in human macrophages and dendritic cells and induce IL-1 $\alpha / \beta$ [10]. However, the contribution of CCs to inflammation and necrosis in CCE remains obscure to date, mostly owing to the lack of suitable animal models of the disease. Nevertheless, researchers have tried to mimic atheroembolic renal diseases in rabbits and rats by injecting human atherosclerotic material or CCs $[11,12]$. These studies identified early neutrophilic reactions followed by recruitment of macrophages in response to the atherosclerotic material or CCs. All these inflammatory events culminate in the formation of giant cell granulomas in the lumen, luminal thrombosis, and long-term obstruction because of crystal emboli or fibrotic narrowing of the lumen leading to downstream ischemic injury in the kidneys.

\section{Type 2 Crystalline Nephropathy: Intratubular Crystal Formation}

The glomerular ultrafiltrate can be enriched with minerals, proteins, or drug metabolites, especially in states of volume depletion fostering supersaturation. Acute supersaturation can induce a sudden onset of crystal formation, leading to acute episodes of crystal-induced tubular cell injury, interstitial inflammation, and impairment of renal function, i.e., acute kidney injury (AKI) (Table 1). In contrast, long-standing moderate supersaturation can rather promote crystal formation over extended periods of time, tubule obstruction, and tissue remodeling, i.e., chronic kidney disease (CKD) (Table 1). For example, monoclonal gammopathies can be associated with accelerated filtration of immunoglobulin light chains, some of which form crystals inside the lumen of the proximal tubules, depending on their specific physiochemical properties [13]. Other options are precipitation as $\beta$-sheets and amyloid fibril deposition often leading to nephrotic syndrome $[13,14]$. Occasionally, light-chain crystals also precipitate in intrarenal mononuclear phagocytes causing so-called crystalglobulinemia or crystal-storing histiocytosis $[13,14]$. Chemotherapy for leukemia can induce tumor lysis syndrome, a condition characterized by uric acid release from dying blood cells and monosodium urate (MSU) crystal precipitates inside the tubular lumen. 
Obstruction of distal tubules and collecting ducts lead to AKI, a condition addressed by hydration, alkalization, and preventive uric acid breakdown with recombinant uricase, an enzyme clearing the metabolite substrate of such crystal plugs.

Patients with advanced liver disease retain high levels of bile salts in the circulation as clinically evident by severe jaundice. Once filtered in the kidney, bile salts can precipitate and form casts that obstruct nephrons. In addition, bile salts and bilirubin elicit direct toxic effects on renal tubular cells. Bile cast nephropathy can be a contributing factor in so-called "hepatorenal syndrome" [15]. Other gastrointestinal disorders such as celiac disease, inflammatory bowel disease, or a previous intestinal bypass surgery can be associated with oxalate nephropathy [16]. All of these conditions are associated with increased intestinal oxalate absorption implying hyperoxalemia and hyperoxaluria. Inside the nephron, oxalate does not crystallize up to the distal tubule, the site of calcium secretion. Microcrystal formation, crystal adhesion to the luminal tubular cell membranes, and crystal growth can affect nephron function and cause AKI or CKD [16]. Nephrocalcinosis is also a manifestation of numerous rare genetic metabolic or renal disorders as listed in Table $1[1,14,17]$.

Many mechanisms contribute to AKI associated with rhabdomyolysis. Muscle cell injury follows a release of myoglobin that is freely filtered and can precipitate inside renal tubules, especially during hypovolemia, often coinciding in earthquake or car crash victims [18]. Dehydration is also a common cofactor in crystal-induced AKI related to exposures to drugs, toxins, or dietary components that tend to crystallize in the urine. Oxalate-rich food products as a cause of secondary hyperoxaluria and acute oxalate nephropathy include spinach, nuts, black tea, rhubarb, and certain fruits, especially when intestinal abnormalities increase oxalate uptake. Vitamin C supplements or accidental polyethylene glycol ingestion trigger acute oxalate nephropathy because oxalate is their major downstream water-soluble metabolite $[19,20]$. Sodium phosphate, a component of oral bowel purgatives often used before colonoscopy, can cause massive intratubular precipitation of calcium phosphate crystals, leading to acute phosphate nephropathy [21]. Finally, sulfamethoxazole, indinavir, aciclovir, and other drugs can cause acute crystalline kidney injury, especially when high doses coincide with low fluid intake [22].

\section{Pathophysiology of AKI}

Supersaturation of urine because of either dehydration-induced concentration or extensive exposure to crystal-forming substances results in crystallization of solutes in renal tubules [20]. These deposited crystals then contribute to kidney injury by inducing direct and indirect cytotoxicity as well as by setting up an auto-amplification loop of necroinflammation [23-25].

Direct Crystal Cytotoxicity. Although tubular cells surrounding crystal deposits often die by necrosis, the underlying mechanisms of such cytotoxicity effect remained ambiguous. It has been proposed that tubular cells try to digest smaller crystals in lysosomes after phagocytosis. During this process, calcium-containing crystals release free calcium into the cytosol and thus engage the calpainmediated cellular necrosis pathways $[1,2]$. Conversely, indigestible lysosomal crystals can destabilize lysosomal membranes, release lysosomal enzymes into cytosol causing cell stress to engage autophagic tubular cell death. Some of the lysosomal enzymes - e.g., cathepsin B - are reported to activate necroptosis by cleaving the necroptosis inhibitor protein RIPK1 in immune cells [26]. In fact, crystals of calcium oxalate, calcium phosphate, calcium pyrophosphate, cysteine, cholesterol, and MSU induced tubular cell necroptosis in vitro by engaging RIPK3 and pseudokinase MLKL [5, 6] (Fig. 2). Moreover, both chemical inhibition as well as deficiency of promotors of necroptosis - for example, Ripk3 and Mlkl deficiency protected mice from acute oxalate nephropathy [5]. On the contrary, deficiency of inhibitors of necroptosis - for example, repulsive guidance molecule b aggravated acute oxalate nephropathy [27]. During rhabdomyolysis-induced AKI, tubular cytotoxicity because of heme crystals also involved the RIPK3-MLKL pathway. In addition, oxalate and folic acid crystals induced ferroptosis of tubular cells in vivo, resulting in AKI $[28,29]$. However, the ability of oxalate and folic acid crystals to induce ferroptosis in vitro remains obscure to date.

Indirect Crystal Cytotoxicity. Crystals can also trigger necrosis of tubular cells indirectly via necroinflammation [23] (Fig. 2). Crystal-induced cellular necrosis leads to the release of danger-associated molecular patterns, highmobility group protein 1 , histones, mitochondrial DNA, demethylated DNA and RNA, ATP, uric acid, and double-stranded DNA into the extracellular compartment [23]. These factors may induce regulated necrosis of neighboring cells by engaging death receptors on their surface $[7,23]$. Moreover, because of their strong basic charge, extracellular histones disrupt plasma membranes of neighboring live cells and thus aggravate renal injury [30]. Furthermore, a broad range of crystals of different size and shape are reported to induce neutrophil necroptosis [31, 32]; however, whether crystal-induced neutro- 
Fig. 2. Molecular mechanisms of crystal-induced necroinflammation. Renal cells try to digest crystals deposited in kidneys in phagolysosomes after engulfing them by phagocytosis. This results in $\mathrm{Ca}^{2+}$ release in the cytosol and subsequent activation of calpains leading to IL-1a release, which sets up inflammation. However, bigger and indigestible crystals induce lysosomal destabilization and thereby release cathepsin B in the cytosol. Cathepsin B and reactive oxygen species activate the NACHT, LRR, and PYD domains containing protein 3 (NLRP3) inflammasome and cause secretion of mature IL-1 $\beta$. Cathepsin B also cleaves RIPK1, which leads to either caspase-mediated apoptosis or RIPK3- and MLKL-mediated cellular necroptosis in the absence of caspase 8. Dying renal cells release various DAMPs and alarmins that contribute to renal inflammation. Certain pro-inflammatory cytokines can also induce regulated cell death in neighboring live cells and thus set up an auto-amplification loop of necroinflammation. DAMPs, danger-associated molecular patterns; MLKL, mixed lineage kinase domain-like; RIPK1, receptor-interacting serine/threonine-protein kinase 1; RIPK3, receptor-interacting serine/threonine-protein kinase 3.

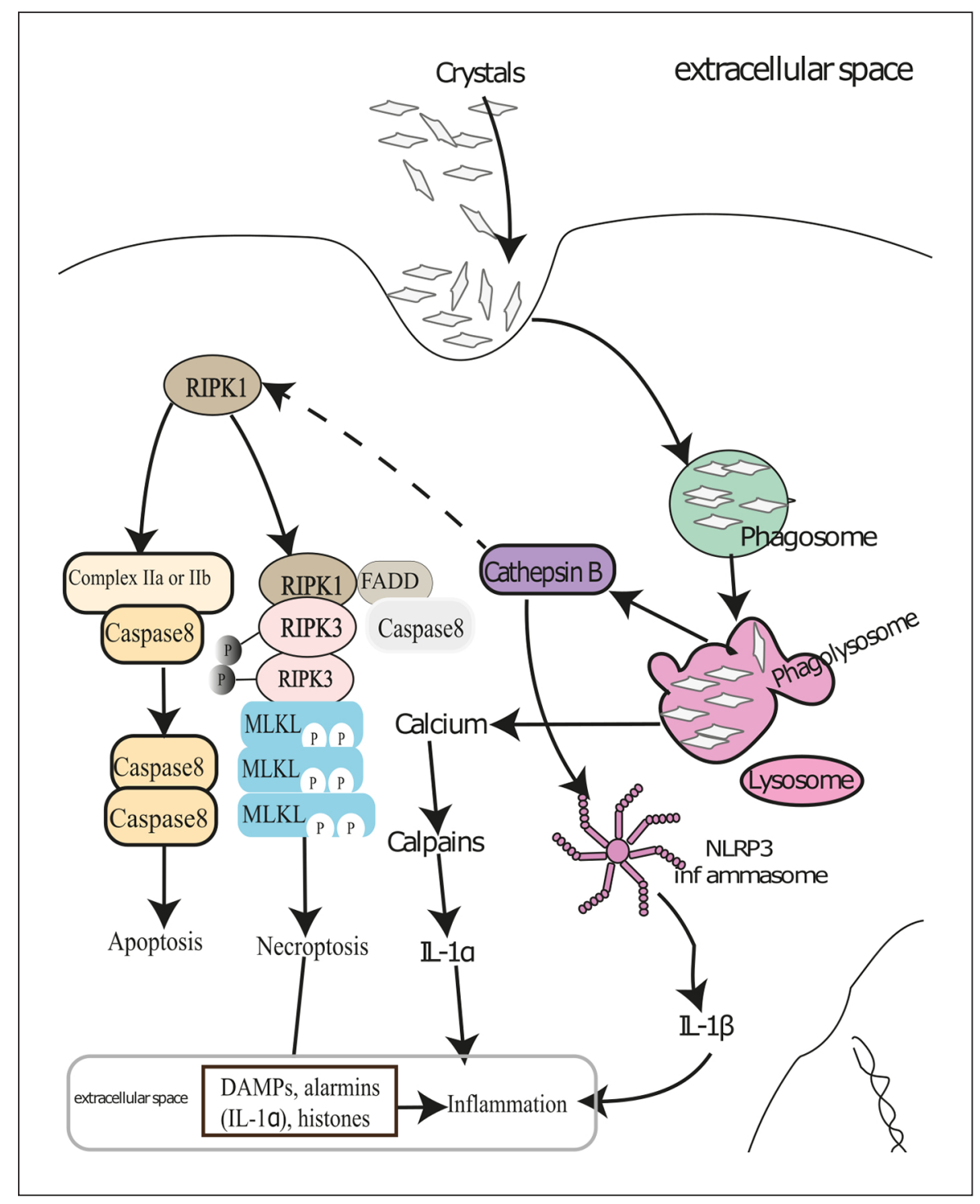

phil necroptosis contributes to crystalline nephropathies remains unexplored to date.

Crystal-Induced Renal Inflammation. As mentioned earlier, crystal-induced cellular necrosis leads to the release of danger-associated molecular patterns, alarmins, histones, proteases, etc., which can also trigger inflammation via activating the Toll-like receptors (TLRs). Tubular cell necrosis promotes leukocyte influx in the kidney as well as complement activation, thus enhancing renal necroinflammation [24]. In addition, crystals also activate the NLRP3 inflammasome in renal phagocytes and induce secretion of mature IL- $1 \beta$ during crystalline nephropathy [33]. A variety of crystals share similar mechanisms of NLRP3 inflammasome activation - e.g., phago- cytosis, reactive oxygen species production, cathepsin B release, and potassium efflux $[33,34]$. Further, mature IL- $1 \beta$ sets up subsequent inflammation via the IL- 1 receptor [33]. Some crystals can also induce inflammation via other pathways in addition to inflammasome activation. For example, direct membrane binding of MSU crystals induces cell surface lipid sorting and activation of a tyrosine protein kinase Syk in a receptor-independent manner [34].

Activation of NLRP3 inflammasomes induces cell death, which is termed pyroptosis [7]. Although crystals do not induce pyroptosis of tubular cells directly $[5,6]$, the chances of tubular cell death via pyroptosis cannot be ignored in crystalline nephropathies. Some inflamma- 
tory cytokines (e.g., TNFa) drive necroptosis of tubular cells to enhance oxalate crystal-induced AKI [5]. Multiple cathepsins are also suggested to regulate crystal-induced cell death in an inflammasome-independent manner [35]. Furthermore, physical obstruction of the tubular lumen by crystals themselves or necrotic cell debris contributes to a decline in renal function in crystalline nephropathies [1]. All these processes constitute the auto-amplification loop of necroinflammation [7, 23]. Interestingly, an inhibitory C-type lectin receptor (Clec12a) was recently identified as an inhibitory receptor for MSU that regulates inflammation in response to cell death [36], though its contribution to the suppression of crystal-induced renal inflammation remains to be studied.

\section{Pathophysiology of CKD}

Crystalline $\mathrm{CKD}$, also referred to as kidney stone disease, if often a result of genetic disorders that enhance the synthesis of certain minerals or metabolites so much that their concentration in urine reaches supersaturation, leading to their crystallization (Table 1). The term nephrocalcinosis refers only to the presence of calcium phosphate and calcium oxalate crystals in the kidney. However, kidney stones comprise crystals of uric acid and adenine, in addition to calcium-containing crystals.

Sites of Intrarenal Crystallization. (a) The proximal tubule. It is the most preferred site of crystallization in the kidney owing to its native function. Proximal tubules reabsorb most of the glomerular filtrate, and thus, concentrate urine, which favors supersaturation and crystallization of minerals, metabolites, small proteins such as light chains, etc. (b) The thick ascending loop of Henle. It reabsorbs chloride ions from the urine, leading to urine acidification, favoring crystallization of lithogenic solutes such as uric acid, and secretes calcium ions into urine, favoring crystallization of calcium-containing solutes such as calcium phosphate and oxalate. In addition, it secretes uromodulin, which enhances cast formation in association with light chains, necrotic debris, as well as myoglobin [1]. (c) The medullary collecting ducts. They passively excrete urea in the urine, and therefore, are the preferential sites of uric acid crystallization in the kidney [37]. (d) The interstitial compartment. Extratubulation of intratubular crystal plugs leads to crystal granuloma in the renal interstitial compartment [38]. The presence of crystal granulomas is a characteristic feature of certain forms of crystalline CKD, e.g., urate nephropathy.

Crystal-Induced Kidney Injury
Mechanisms of Crystal-Induced Inflammation. Similar to crystalline AKI, crystal deposits in crystalline CKD also activate the NLRP3 inflammasome and induce inflammation in the kidney. Deficiency of inflammasome components, NLRP3, and ASC in mice reduced renal inflammation during progressive renal failure in nephrocalcinosis-related CKD [39, 40]. Furthermore, chemical inhibition of NLRP3 using a specific inhibitor - CP456773 or $\beta$-hydroxybutyrate - reduced renal inflammation and progressive decline in renal function in murine nephrocalcinosis-related CKD [40, 41]. Inflammasome activation leads to secretion of mature IL- $1 \beta$ and IL-18 that contribute to renal inflammation. Surprisingly, IL-1 receptor inhibition using anakinra has no effect on progressive decline in renal function in murine nephrocalcinosis-related CKD [40, 41], suggesting a contribution of inflammasome-independent functions of NLRP3, which involve transforming growth factor beta receptor signaling-mediated renal fibrosis [42]. Indeed, chemical inhibition of NLRP 3 by CP-456773 during oxalate and adenine nephropathy or by $\beta$-hydroxybutyrate during oxalate nephropathy reduced intrarenal fibrosis in murine crystalline CKD models $[40,41]$. In addition, our own data demonstrated that $\beta$-hydroxybutyrate induced M2 macrophage phenotype shift and also contributed to a decline in renal inflammation and preserved renal function during chronic oxalate nephropathy without affecting intrarenal crystal deposition [40].

Macrophages, which often have a giant cell-like appearance, border the extratubular crystal plaques in the interstitial compartment, leading to the term "crystal granuloma" [1]. They are thought to contribute to the removal of crystal deposits via phagocytosis and digestion [1]. Consistently, colony-stimulating factor suppressed renal crystal deposition by inducing M2 macrophages in the kidneys of hyperoxaluric mice [43]. However, the molecular mechanisms that trigger the formation of crystal granulomas in crystalline CKD are unknown to date.

Furthermore, the procedure of crystallization itself has immunostimulatory effects - e.g., high concentrations of uric acid activate TLRs (TLR4) and induce expression of inflammasome components [34], whereas oxalate ions induce expression of chemokines and osteopontin, which regulate inflammation [44]. Moreover, crystal plugs adhere to the tubular lumen and cause obstruction, leading to declined renal function. Crystal adhesion to tubular epithelium is regulated by several surface molecules such as CD44, annexin II, osteopontin, phosphatidylserine, hyaluronan, as well as TNF receptor signaling [45-47]. 


\section{Type 3 Crystalline Nephropathy: Urolithiasis}

Nephrolithiasis, ureterolithiasis, or cystolithiasis can cause nephron loss, renal inflammation, and fibrosis as a manifestation of obstructive nephropathy. In the North American population, $7-11 \%$ are effected by urolithiasis, with a preponderance of male individuals; however, CKD as an obvious consequence of urolithiasis is recognized only in a small percentage of stone formers [45]. Many factors contribute to urolithiasis, but hereditary metabolic diseases associated with an increased production of cysteine, uric acid, or oxalate are paradigmatic examples illustrating the pathogenesis of crystalluria and stone formation (Table 1). Variants in genes encoding for mineral or metabolite transporters that promote supersaturation of minerals or metabolites in the urine are another common cause of urolithiasis [17]. In addition, diets rich in fructose or oxalate, together with low fluid intake or excessive sweating or diarrhea, can have synergistic effects on microcrystal and stone formation $[45,48]$.

Urolithiasis most commonly presents as renal colic or a sudden onset of crescendo and decrescendo in flank pain or the inguinal region. Oliguria is absent unless bilateral urolithiasis causes simultaneous obstruction [49]. Stone passage or removal relieves pressure and pain. Most frequently, stones suddenly mobilize from calyces of the renal pelvis, but the triggering events remain largely unknown. During stone passage, the rough surface of the calculus, together with the mechanical forces of ureteric peristalsis, can cause mucosal injury, bleeding, and swelling at the site of obstruction. Despite the alarming clinical presentation, renal colic is not necessarily associated with long-term impairments of urinary tract function unless chronic obstruction leads to obstructive nephropathy and irreversible nephron loss.

Calculus formation often initiates at Randall plaques inside the renal pelvis, i.e., interstitial apatite plaques formed along thin parts of the loops of Henle [46]. Randall plaques are a preferred nidus site for calcium oxalate stones. The plaque-stone interface is a multilayer ribbon consisting of matrix molecules such as Tamm-Horsfall urinary glycoprotein and calcium oxalate crystals. Indeed, although uromodulin inhibits luminal crystal formation, it is a regular component of interstitial crystal plaques. Sites of brushite and hydroxyapatite stone formation in the renal pelvis are plugs inside Bellini ducts and inner medullary collecting ducts formation. Such plugs occur upon ileostomy, small bowel resection, or bariatric surgery as well as cystinuria, primary hyperparathyroidism, or primary hyperoxaluria [50].
In healthy kidneys, crystal nucleation does not necessarily lead to crystal attachment or growth [46]. Indeed, epithelial injury or absence of crystallization inhibitors enhances crystal adhesion to the tubular epithelium [38, 46]. For example, own experiments suggest that the receptors of TNFa mediate the adhesion of calcium oxalate crystals to the tubular epithelium by inducing the expression of crystal adhesion molecules at the luminal surface [47]. However, the process of adhesion and growth of calcium oxalate stones at Randall plaques and plugs obstructing the ducts of Bellini remains poorly understood. Some factors include plug composition, urine flow, and mineral supersaturation $[38,46]$.

\section{Summary}

Crystals contribute to kidney injury involving a broad variety of different pathomechanisms. Vascular manifestations (type 1) involve calcium phosphate deposits inside the vascular wall or CC deposits in atheromatous plaques that both can contribute to renal ischemia by contributing to renal artery stenosis or by cholesterol embolism. Supersaturation of minerals or certain proteins can promote crystallization inside the tubular compartment and cause tubular obstruction and tubular epithelial cell injury up to necrosis. These processes trigger inflammation, interstitial edema, and eventually renal fibrosis (type 2). Nephro- and urolithiasis present as renal colic or obstructive nephropathy, which when chronic can cause extensive nephron damage. The molecular mechanisms that link crystal formation to adhesion, growth, renal inflammation, and renal cell death remain largely to be discovered. There is hope that a better understanding of the underlying pathophysiology will help develop new cures for patients with crystalline nephropathies.

\section{Acknowledgments}

S.R. Mulay and H.J. Anders were supported by the Deutsche Forschungsgemeinschaft (MU 3906/1-1, AN372/16-2, 20-1, and 24-1).

\section{Conflict of Interest Statement}

The authors have no conflicts of interest to disclose. 


\section{References}

$>1$ Mulay SR, Anders HJ: Crystal nephropathies: mechanisms of crystal-induced kidney injury. Nat Rev Nephrol 2017;13:226-240.

2 Mulay SR, Anders HJ: Crystallopathies. N Engl J Med 2016;374:2465-2476.

$>3$ Venturelli C, Jeannin G, Sottini L, Dallera N, Scolari F: Cholesterol crystal embolism (atheroembolism). Heart Int 2006;2:155.

4 Pilely K, et al: Cholesterol crystals activate the lectin complement pathway via ficolin-2 and mannose-binding lectin: implications for the progression of atherosclerosis. J Immunol 2016;196:5064-5074.

7 Mulay SR, et al: Cytotoxicity of crystals involves RIPK3-MLKL-mediated necroptosis. Nat Commun 2016;7:10274.

6 Honarpisheh M, et al: Phagocytosis of environmental or metabolic crystalline particles induces cytotoxicity by triggering necroptosis across a broad range of particle size and shape. Sci Rep 2017;7:15523.

$>7$ Linkermann A, Stockwell BR, Krautwald S, Anders HJ: Regulated cell death and inflammation: an auto-amplification loop causes organ failure. Nat Rev Immunol 2014;14:759767.

$>8$ Duewell P, et al: NLRP3 inflammasomes are required for atherogenesis and activated by cholesterol crystals. Nature 2010;464:13571361.

-9 Kiyotake R, et al: Human Mincle binds to cholesterol crystals and triggers innate immune responses. J Biol Chem 2015;290:2532225332.

$>10$ Corr EM, Cunningham CC, Dunne A: Cholesterol crystals activate Syk and PI3 kinase in human macrophages and dendritic cells. Atherosclerosis 2016;251:197-205.

11 Warren BA, Vales O: Electron microscopy of the sequence of events in the atheroembolic occlusion of cerebral arteries in an animal model. Br J Exp Pathol 1975;56:205-215.

$>12$ Gore I, McCombs HL, Lindquist RL: Observations on the fate of cholesterol emboli. J Atheroscler Res 1964;4:527-535.

$>13$ Doshi M, et al: Paraprotein-related kidney disease: kidney injury from paraproteins what determines the site of injury? Clin J Am Soc Nephrol 2016;11:2288-2294.

14 Motwani SS, et al: Paraprotein-related kidney disease: glomerular diseases associated with paraproteinemias. Clin J Am Soc Nephrol 2016;11:2260-2272.

-15 van Slambrouck CM, Salem F, Meehan SM, Chang A: Bile cast nephropathy is a common pathologic finding for kidney injury associated with severe liver dysfunction. Kidney Int 2013;84:192-197.

16 Robijn S, Hoppe B, Vervaet BA, D’Haese PC, Verhulst A: Hyperoxaluria: a gut-kidney axis? Kidney Int 2011;80:1146-1158.

-17 Sayer JA: Progress in understanding the genetics of calcium-containing nephrolithiasis. J Am Soc Nephrol 2017;28:748-759.
18 Vanholder R, Sever MS, Erek E, Lameire N: Rhabdomyolysis. J Am Soc Nephrol 2000;11: 1553-1561.

19 Morfin J, Chin A: Images in clinical medicine. Urinary calcium oxalate crystals in ethylene glycol intoxication. N Engl J Med 2005;353: e21.

20 Terlinsky AS, Grochowski J, Geoly KL, Strauch BS, Hefter L: Monohydrate calcium oxalate crystalluria in ethylene glycol poisoning. N Engl J Med 1980;302:922.

21 Hoffmanova I, Kraml P, Andel M: Renal risk associated with sodium phosphate medication: safe in healthy individuals, potentially dangerous in others. Expert Opin Drug Saf 2015;14:1097-1110.

22 Hess B: Drug-induced urolithiasis. Curr Opin Urol 1998;8:331-334.

23 Mulay SR, Linkermann A, Anders HJ: Necroinflammation in kidney disease. J Am Soc Nephrol 2016;27:27-39.

24 Mulay SR, Kumar SV, Lech M, Desai J, Anders HJ: How kidney cell death induces renal necroinflammation. Semin Nephrol 2016;36: 162-173.

25 Mulay SR, Holderied A, Kumar SV, Anders $\mathrm{HJ}$ : Targeting Inflammation in so-called acute kidney injury. Semin Nephrol 2016;36: 17-30.

$26 \mathrm{McComb}$, et al: Cathepsins limit macrophage necroptosis through cleavage of Rip1 kinase. J Immunol 2014;192:5671-5678.

27 Liu W, et al: RGMb protects against acute kidney injury by inhibiting tubular cell necroptosis via an MLKL-dependent mechanism. Proc Natl Acad Sci USA 2018;115:E1475-E1484.

28 Martin-Sanchez D, et al: Ferroptosis, but not necroptosis, is important in nephrotoxic folic acid-induced AKI. J Am Soc Nephrol 2017; 28:218-229.

29 Linkermann A, et al: Synchronized renal tubular cell death involves ferroptosis. Proc Natl Acad Sci USA 2014;111:16836-16841.

30 Kumar SV, et al: Neutrophil extracellular trap-related extracellular histones cause vascular necrosis in severe GN. J Am Soc Nephrol 2015;26:2399-2413.

31 Desai J, et al: PMA and crystal-induced neutrophil extracellular trap formation involves RIPK1-RIPK3-MLKL signaling. Eur J Immunol 2016;46:223-229.

32 Desai J, et al: Particles of different sizes and shapes induce neutrophil necroptosis followed by the release of neutrophil extracellular trap-like chromatin. Sci Rep 2017;7:15003.

33 Mulay SR, et al: Calcium oxalate crystals in duce renal inflammation by NLRP3-mediated IL-1 $\beta$ secretion. J Clin Invest 2013;123: 236-246.

34 Franklin BS, Mangan MS, Latz E: Crystal formation in inflammation. Annu Rev Immunol 2016;34:173-202.
35 Orlowski GM, et al: Frontline science: multiple cathepsins promote inflammasome-independent, particle-induced cell death during NLRP3-dependent IL-1 $\beta$ activation. J Leukoc Biol 2017;102:7-17.

36 Neumann K, et al: Clec12a is an inhibitory receptor for uric acid crystals that regulates inflammation in response to cell death. Immunity 2014;40:389-399.

37 Noda S: On mechanism of urate kidney stone formation. Scan Electron Microsc 1984(Pt 4): 1801-1808.

38 Vervaet BA, Verhulst A, D’Haese PC, De Broe ME: Nephrocalcinosis: new insights into mechanisms and consequences. Nephrol Dial Transplant 2009;24:2030-2035.

-39 Knauf F, et al: NALP3-mediated inflammation is a principal cause of progressive renal failure in oxalate nephropathy. Kidney Int 2013;84:895-901.

40 Anders HJ, et al: The macrophage phenotype and inflammasome component NLRP3 contributes to nephrocalcinosis-related chronic kidney disease independent from IL-1-mediated tissue injury. Kidney Int 2018;93:656669

41 Ludwig-Portugall I, et al: An NLRP3-specific inflammasome inhibitor attenuates crystalinduced kidney fibrosis in mice. Kidney Int 2016;90:525-539.

42 Lorenz G, Darisipudi MN, Anders HJ: Canonical and non-canonical effects of the NLRP3 inflammasome in kidney inflammation and fibrosis. Nephrol Dial Transplant 2014;29:41-48.

43 Taguchi K, et al: Colony-stimulating factor-1 signaling suppresses renal crystal formation. J Am Soc Nephrol 2014;25:1680-1697.

44 Umekawa T, Chegini N, Khan SR: Oxalate ions and calcium oxalate crystals stimulate MCP-1 expression by renal epithelial cells. Kidney Int 2002;61:105-112.

-45 Worcester EM, Coe FL: Clinical practice. Calcium kidney stones. N Engl J Med 2010;363: 954-963.

46 Randall A: The origin and growth of renal calculi. Ann Surg 1937;105:1009-1027.

47 Mulay SR, et al: Hyperoxaluria requires TNF receptors to initiate crystal adhesion and kidney stone disease. J Am Soc Nephrol 2017;28: 761-768.

48 Roncal-Jimenez C, et al: Heat stress nephropathy from exercise-induced uric acid crystalluria: a perspective on Mesoamerican nephropathy. Am J Kidney Dis 2016;67:20-30.

49 Miller DC, Wolf JS Jr: Acute renal colic. N Engl J Med 2004;350:2422-2423; author reply 2422-2423.

50 Coe FL, Worcester EM, Evan AP: Idiopathic hypercalciuria and formation of calcium renal stones. Nat Rev Nephrol 2016;12:519533. 